\title{
Familiar-face recognition and comparison: source analysis of scalp-recorded event-related potentials
}

\author{
Elena V. Mnatsakanian ${ }^{\mathrm{a}, \mathrm{b}, *}$, Ina M. Tarkka ${ }^{\mathrm{a}}$ \\ ${ }^{\mathrm{a}}$ Brain Research and Rehabilitation Center Neuron, Kortejoki, FIN-71130 Kuopio, Finland \\ ${ }^{\mathrm{b}}$ Institute of Higher Nervous Activity and Neurophysiology, Russian Academy of Sciences, 5a Butlerova str., Moscow 117485, Russia
}

Accepted 25 November 2003

\begin{abstract}
Objective: We studied the event-related potentials elicited by categorical matching of faces. The purpose was to find cortical sources responsible for face recognition and comparison.

Methods: Nineteen healthy volunteers participated in the study. Each trial began with one of the two cues (S1) followed by consecutive pictures (S2 and S3). Each picture was a photograph of a familiar face with a superimposed abstract dot pattern. One cue directed attention to compare faces and another to compare patterns. 128-channel electroencephalogram was recorded. Spatio-temporal multiple dipole source models were generated using Brain Electromagnetic Source Analysis 2000, for the window of 80-600 ms from S3 onset.

Results: The obtained model for face recognition and comparison contained 8 dipoles explaining $97 \%$ of grand average and about $90 \%$ of individual data and showing temporal and spatial separation of sources: in the frontal region, in the occipital cortex, and in the bilateral medial temporal and inferotemporal regions. Different faces elicited larger components than same person's faces around $400 \mathrm{~ms}$, mainly explained by frontal dipoles.
\end{abstract}

Conclusions: The sources in our models estimate the activity common for both Face task conditions (the recognition of a familiar person) and also differential activity, related to the match/mismatch item processing.

(C) 2004 International Federation of Clinical Neurophysiology. Published by Elsevier Ireland Ltd. All rights reserved.

Keywords: N400-like components; Priming; Multiple dipole model; Face processing; Eelectroencephalogram

\section{Introduction}

Human faces are very relevant, affective, and socially meaningful visual objects. Cerebral responses elicited by faces differ from those elicited by other kinds of visual objects (Allison et al., 1994; Bentin et al., 1996). According to the well-known model for face processing (Bruce and Young, 1986), after the initial stage of structural encoding, faces are further analyzed for the expression, age, etc. The component N170 (N200 or vertex-positive potential, VPP, in some papers) presumably corresponds to the structural encoding stage and has its origin in temporal regions (Allison et al., 1994; Haxby et al., 2000). Familiar faces trigger parallel analysis of personal identity, which may be related to components with latencies around 400-600 ms (Eimer, 2000). At the same latencies, the phenomena of priming (or expectancy, or congruity) were first described in

\footnotetext{
* Corresponding author. Tel.: + 358-17-460-281; fax: + 358-17-460-200

E-mail address: mnazak@aha.ru (E.V. Mnatsakanian).
}

verbal tasks (Kutas and Hillyard, 1980) and later found also for non-linguistic stimuli (Jemel et al., 1999; McPherson and Holcomb, 1999; Munte et al., 1998). There is evidence for multiple components at these latencies: N300 and N400 for pictures (McPherson and Holcomb, 1999; Barrett and Rugg, 1990), and N350 and N380 for faces (Jemel et al., 1999).

The purpose of this study was to determine the locations and temporal dynamics of brain sources for the evoked electrical activity during the processing of familiar faces. This was done by developing multiple dipole source models for scalp-recorded event-related potentials (ERPs). Some aspects of face processing have been explained by dipole models elsewhere (Jemel et al., 1999; Rossion et al., 1999; Watanabe et al., 1999). Even though the non-unique nature of a solution of the inverse problem is well known, the dipolar source localization can reduce the amount of data and provide relatively robust active regions and their temporal relations during the information processing. 


\section{Methods}

\subsection{Participants}

Nineteen healthy volunteers (13 females and 6 males) aged from 29 to 52 years (with an average of 42 years) participated in this study. They were right-handed and had normal or corrected-to-normal binocular visual acuity. They were personnel of a hospital, had no history of neurological disorders, were non-medicated, and did not receive any payment for their participation. The experiment was approved by the local ethical committee, and all participants provided an informed consent.

\subsection{Stimuli}

Each of the two cue stimuli (S1) was a figure of white dots on a gray background, and S2 and S3 stimuli consisted of superimposed faces and dot patterns (Fig. 1b). The faces were black and white photographs of 11 persons familiar to our participants (colleagues). Each person was represented with two portraits both having a neutral facial expression. The patterns include 15 abstract figures made of white dots. Each visual stimulus $(13.6 \times 10.6 \mathrm{~cm})$ was exposed for $250 \mathrm{~ms}$ at a distance of $1.7 \mathrm{~m}$.

\subsection{Procedure}

The experiments consisted of a total of 200 trials given in 4 series with short resting periods between the series. The exact timing of a single trial is shown in Fig. 1A. Each trial began with one of the two cues (with equal probability) followed by the $S 2-S 3$ pair. Since both $S 2$ and $S 3$ consisted of superimposed faces and patterns, the choice of the item to compare depended on the cue (S1). After seeing one of the cues, the participants had to compare faces (Face task), and after another cue they had to compare patterns (Pattern task). For both tasks, the targets were the same or different pairs with equal probability. In the Same condition of the Face task, different views of the same person were always presented (categorical matching of faces).

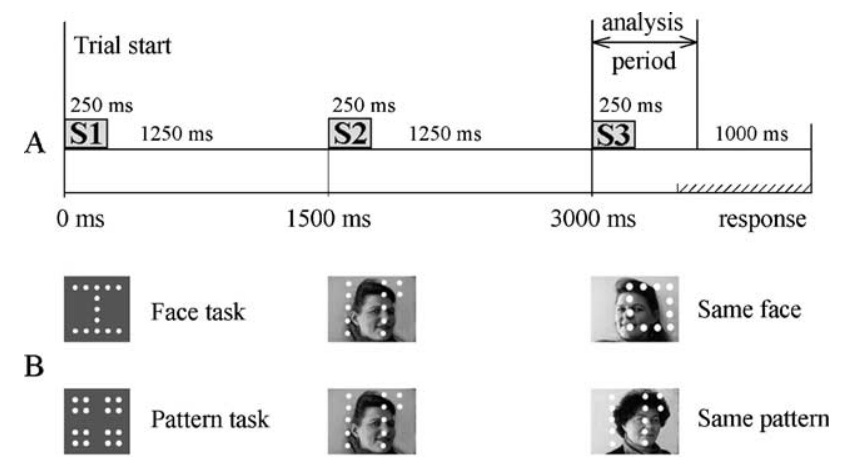

Fig. 1. (A) The progression of a single trial from 0 to $4000 \mathrm{~ms}$. (B) The cues and the samples of picture stimuli are shown with the instructions for cues and the correct responses.
After S3, participants were to judge whether they had seen the same target as in S2. The participants were instructed to press the keys on the response pad with index (same items) and middle (different items) fingers of their right hand. The intertrial interval $(750 \mathrm{~ms})$ began from the participant's response or, if there was no or late response, from the end of the waiting period $(1250 \mathrm{~ms}$ from the $\mathrm{S} 3$ onset). We report the results for a period of $600 \mathrm{~ms}$ from the onset of the second picture in a pair (S3) for the Face task.

\subsection{Data acquisition and preprocessing}

Data were acquired with a system designed by Electrical Geodesics, Inc. (Eugene, OR, USA). Electroencephalogram (EEG) was recorded with a 128-electrode net using $\mathrm{Cz}$ as the reference site. The sampling rate was $250 \mathrm{~Hz}$, with filters of $0.01-100 \mathrm{~Hz}$. EEG was segmented and averaged off-line separately for each condition of both tasks: Same Face (SF) and Different Face (DF); Same Pattern (SP) and Different Pattern (DP). The artifact-free trials with correct responses were averaged, digitally filtered in $0.3-15 \mathrm{~Hz}$, and baselinecorrected. The re-referencing of the original recording was done off-line during the analysis: the $\mathrm{Cz}$-reference montage was changed to the average reference.

\subsection{Dipole source analysis}

Non-invasive source localization was performed with the Brain Electromagnetic Source Analysis (BESA 2000, version 4.2) algorithm (Berg and Scherg, 1994). In the spatio-temporal model, the dipole source has a stationary location and orientation, and changes the moment (i.e. its strength) with time. The source waveform (SWF) describes the temporal changes of the dipole moment of a single source. The temporal separation of the activity of several brain sources can be observed in SWFs as separate components with different peak latencies for each source. The residual variance (RV) describes the proportion of the recorded data that is not explained by the model.

A 4-shell ellipsoidal head model was used to model the brain activity in the $80-600 \mathrm{~ms}$ time window from the S3 onset. To determine the number and the starting locations of dipoles, we performed a principal component analysis, generated current source density maps, and considered the physiological feasibility of sources. The dipole models were developed first for the grand averaged ERP, using an 88-mm head radius (the mean of 19 individual radii; besides, 9 participants had this head radius). During the modeling, only the RV and energy constraints were used as fit criteria (with weighting factors equal to 1), no restrictions for the minimal distance between the sources or the bilateral symmetry were applied. The regularization constant (a parameter used to reduce the interaction between sources) was set to $1 \%$. Several models including the 6-8 dipoles were considered. The RV was low already with a 6-dipole model. However, the temporal separation was not yet 
satisfactory for the earlier components, and the shorter window of $80-300 \mathrm{~ms}$ was used to model these components. Then the locations obtained in this modeling were used to model the whole window. The models with the lowest RV and best temporal separation of components for DF and SF were chosen and used for further analysis: the locations and orientations of dipoles were the same ('base model'), and only the SWFs differed. The locations and orientations of dipoles from this 'base model' were applied to individual data without fitting to obtain the sets of individual SWFs for each dipole. When analyzing the individual data, the dipole coordinates were proportionally adjusted for smaller and larger head sizes. After the initial introduction of the base model to the individual data, only the orientation fitting was performed. The angles were calculated between the orientation in the base model and the orientation obtained after the individual fitting.

To find group differences between DF and SF conditions, the obtained SWFs for the individual data were compared for each dipole using the non-parametric Wilcoxon matched pairs test. The amplitude values were analyzed at each time point of the SWFs for each dipole. Each test included 19 pairs of individual amplitudes (one pair = one participant) at identical time points of SWFs for the corresponding dipoles from each model. The two-tailed $95 \%$ probability was chosen to be the significance threshold.

The base model was also applied to the data from Pattern task to check how this model may explain different visual task. The same statistical analysis was done for the obtained SWFs in Pattern task as described above.

\section{Results}

\subsection{Behavioral performance and ERPs}

The performance rates seemed to be higher for nonmatching faces $(85.1 \pm 3.8 \%$, mean \pm standard error) than for matching faces $(83.4 \pm 4.9 \%)$. The reaction times to matching faces were shorter than to non-matching ones (766 \pm 34 vs. $829 \pm 31 \mathrm{~ms})$. A detailed statistical analysis of the scalp-recorded ERPs and behavioral performance was done in our previous paper for both Face and Pattern tasks (Mnatsakanian and Tarkka, 2003). The grand average data with common average reference are shown in Fig. 2A for 9 of the 128 recording sites for Face task. The grand average ERPs for two most representative channels are shown in Fig. 2B for Pattern task. The largest differences were seen

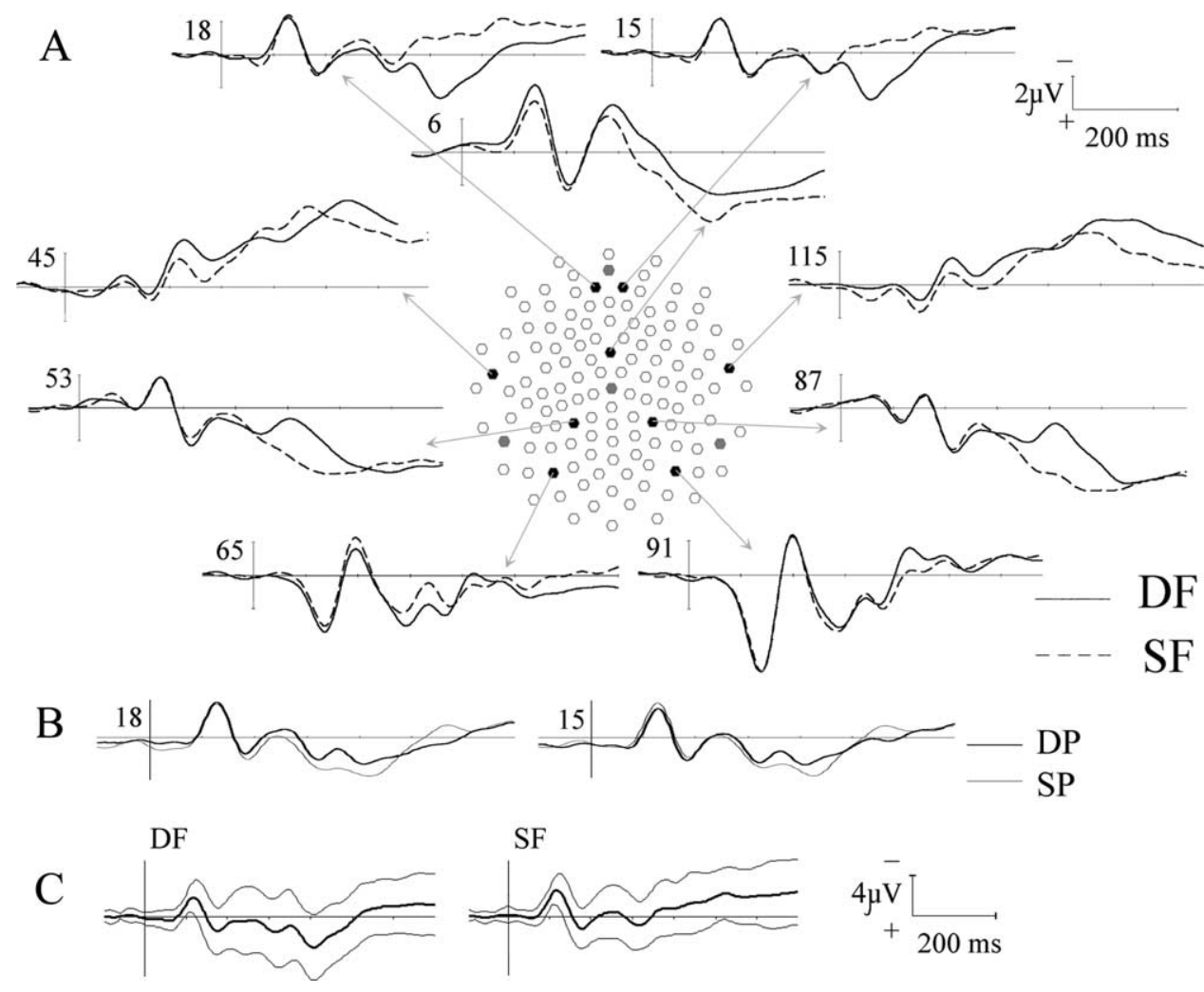

Fig. 2. (A) Grand-averaged ERPs for Different Face (DF) vs. Same Face (SF) conditions are plotted superimposed for each of the selected channels with common average reference. The channels approximately correspond to those of the 10-20 system: 18 (Fp1), 15 (Fp2), 45 (T3), 115 (T4), 6 (FCz), 53 (P3), 87 (P4), 65 (T5), and 91 (T6). Their locations (black dots) are shown in the 128-channel electrode montage. The nose is shown up. The mastoids, Cz, and ground electrodes are indicated with gray dots. The window is $-100+700 \mathrm{~ms}$ from the S3 onset (vertical line). (B) Grand-averaged ERPs for Different Pattern (DP) vs. Same Pattern (SP) conditions are plotted superimposed for channels 15 and 18. Time and amplitude scales, and the time window are as in (A). (C) Grandaverage ERPs (solid line) from channel 15 are shown with the standard deviations (thin lines) for both conditions. The time window is the same as in (A). 
around $400 \mathrm{~ms}$. The grand averages for the right prefrontal site with their standard deviations are shown in Fig. 2C separately to demonstrate that the individual data dispersion seems to be similar for both conditions.

\subsection{Dipole models}

The final models consisted of 8 dipolar sources that differed only in their activity for the DF and SF conditions; the locations and orientations were identical (Fig. 3A). The dipole locations in the Talairach coordinate system (Talairach and Tournoux, 1988) and corresponding anatomical structures are given in Table 1. The projections of the sources on the brain slices from the Talairach Atlas are shown in Fig. 4. The point-like source describes the activity of the named structure and/or the integrated activity from several neighbor structures representing 'the center of mass' of the active area.

The dynamics of source activity as SWF graphs showed a clear separation of the components (Fig. 3B). The components with latencies shorter than $250 \mathrm{~ms}$ were explained mainly by the dipoles $5-8$, and those with longer latencies were generated by the sources $1-4$. Although no symmetry constraint was applied during the modeling, the dipoles 5-6 and 7-8 showed a surprisingly good bilateral symmetry in their locations (less obvious in dipoles 3 and 4). Despite spatial symmetry, the differences in the source activation were obviously hemispheric. The component at $120 \mathrm{~ms}$ (dipole 6) appeared to have a larger amplitude and the component at 150-170 ms (dipole 8) appeared to have a shorter peak latency in the right hemisphere as compared to the left (dipoles 5 and 7 correspondingly). The component at $180 \mathrm{~ms}$ (dipoles 5-6) was quite symmetric in amplitude. Dipole 4 in the right hemisphere seemed to have a smaller amplitude in 200-300 ms than its counterpart in the left hemisphere (dipole 3). This dipole was located more inferior than dipole 3, closer to occipital sources, and the activity at these latencies seems to be partially explained also by dipole 6 , as can be seen from the SWFs. This may be indicative of an active occipital-temporal area in the right hemisphere that is larger and has more complex configuration than in the left.

The sources 1 and 2 were both located close to the midline. We did not succeed to separate these activities into bilaterally symmetric sources by adding more dipoles. Nevertheless, those may be bilateral active spots with left or right preponderance. The source 1 (in the anterior part of cingulate gyrus) was active mostly after $300 \mathrm{~ms}$. The waveforms had blurred maxima at $450 \mathrm{~ms}$ for SF and at 400 and $470 \mathrm{~ms}$ for DF. For dipole 2 (that was close to
A

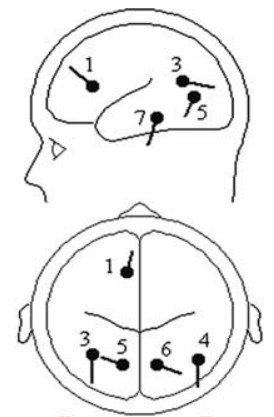

$\mathrm{L}$

$\mathrm{R}$

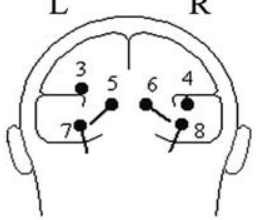

$\mathrm{C}$

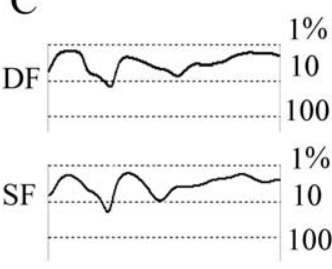

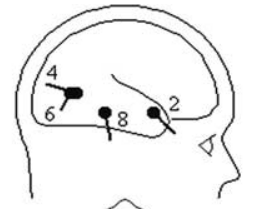

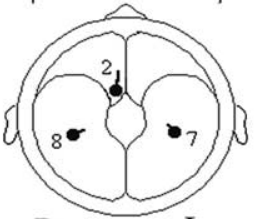

$\mathrm{R}$

L

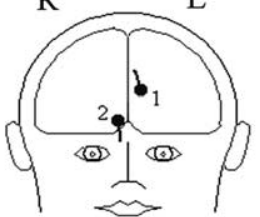

$\mathrm{D}$

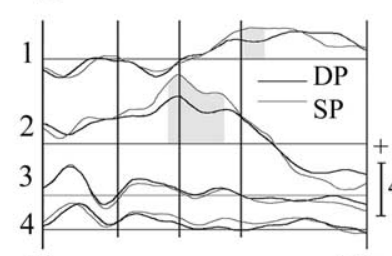

$80 \mathrm{~ms}$

B

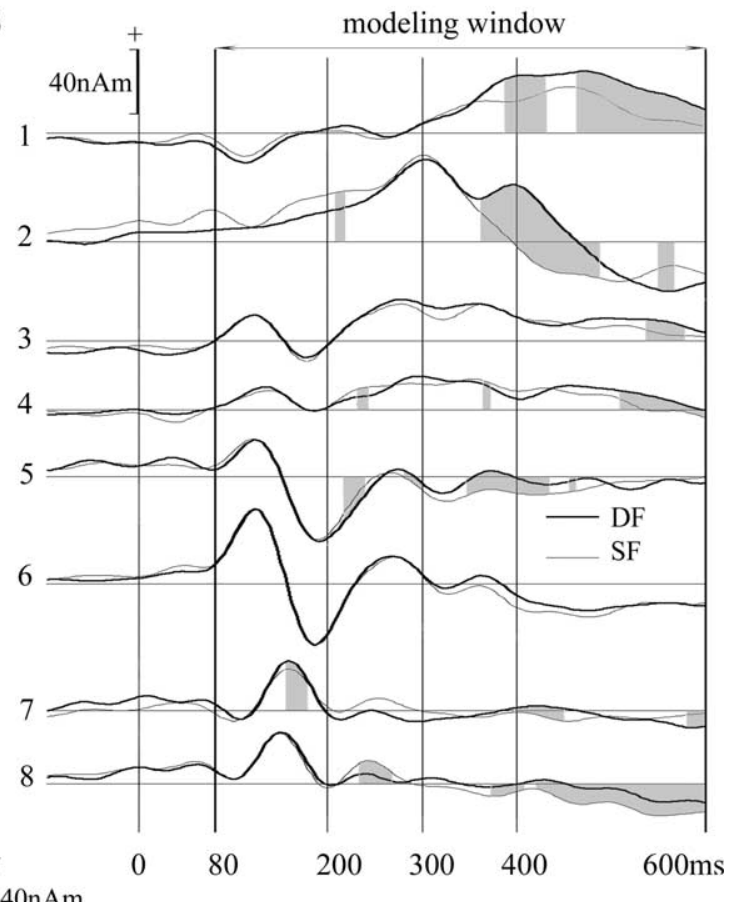

Fig. 3. (A) Dipole locations and orientations in the base model. Transparency in head display is 50\%. (B) Source waveforms for the grand-average data for DF (solid line) and SF (thin line) are shown superimposed for the window of $-100+600 \mathrm{~ms}$ from the S3 onset. The significant group differences are marked in gray color. (C) The temporal dynamics of RVs of the models for the DF and SF conditions are presented on the logarithmic scale from 1 to $100 \%$. (D) Source waveforms for the Pattern task data (DP - solid line and SP - thin line) are shown superimposed for the window of 80-600 ms from the S3 onset with their statistical differences marked in gray color. 
Table 1

The locations of dipoles in the Talairach coordinate system

\begin{tabular}{lrrrll}
\hline$N$ & \multicolumn{2}{l}{$\begin{array}{l}\text { Talairach coordinates } \\
(\mathrm{mm})\end{array}$} & & Anatomical structures & Hemi-sphere \\
\cline { 2 - 4 } & $\mathrm{X}$ & $\mathrm{Y}$ & $\mathrm{Z}$ & & \\
\cline { 5 - 6 } 1 & -10 & 22.9 & 24 & Cingulate gyrus (anterior) & Left \\
2 & 7.6 & 9.2 & -4.9 & Caudate nucleus & Right \\
3 & -36.6 & -58.1 & 19.6 & Medial temporal gyrus & Left \\
4 & 45.9 & -62.8 & 4.4 & Medial temporal gyrus & Right \\
5 & -13.3 & -67.7 & 4 & Lingual gyrus & Left \\
6 & 13.7 & -67.9 & 4 & Lingual gyrus & Right \\
7 & -37.6 & -31.8 & -11.1 & Fusiform/hippocampal gyri & Left \\
8 & 41.4 & -34.9 & -10 & Fusiform/hippocampal gyri & Right \\
\hline
\end{tabular}

the caudate nucleus), the activity peak was observed at $300 \mathrm{~ms}$, and the second peak was observed at $400 \mathrm{~ms}$, this latter only in DF. The largest inter-condition differences were seen in the dipoles 1 and 2 after 350-380 ms. Significant differences at the late latencies were observed also in other sources, with longer durations by those in dipole 8 (right IT cortex) and in dipole 5 (left occipital cortex). The earlier match-mismatch differences were in the dipoles $2,4,5$, and 8 in 200-250 ms; and in the dipole 7 (left IT cortex) in 150-180 ms.
The RV for grand-average data was $2.97 \%$ for DF and $3.21 \%$ for SF conditions. The temporal dynamics of RV in the modeling window is shown in Fig. 3c. The individual $\mathrm{RVs}$ were $11.3 \pm 0.9 \%$ for $\mathrm{DF}$ and $12.3 \pm 1.3 \%$ for $\mathrm{SF}$ (mean \pm standard error). There was no group SF-DF difference for RV values.

We performed also fitting of orientations for each individual model to see if it might improve the modeling results. The fitting of orientations of each individual model decreased the RV values by about $2 \%$. The angles between the dipole orientation in the base model and in each individual model were calculated for each source separately. There were no significant differences in the orientations between the DF and SF conditions other than in dipole $6(P<0.05)$. It seems that, following our strategy, the individual orientation fitting did not add much to the base model of the face processing.

The application of the base model to Pattern task data showed that the base model can also explain other visual tasks. The grand average and individual RV values were in the same range as for Face task. The SWFs for the dipoles 5-8 were very similar to those obtained in Face task; this can be explained by the physical similarity of the stimuli (Fig. 1B). The between-task differences were seen in
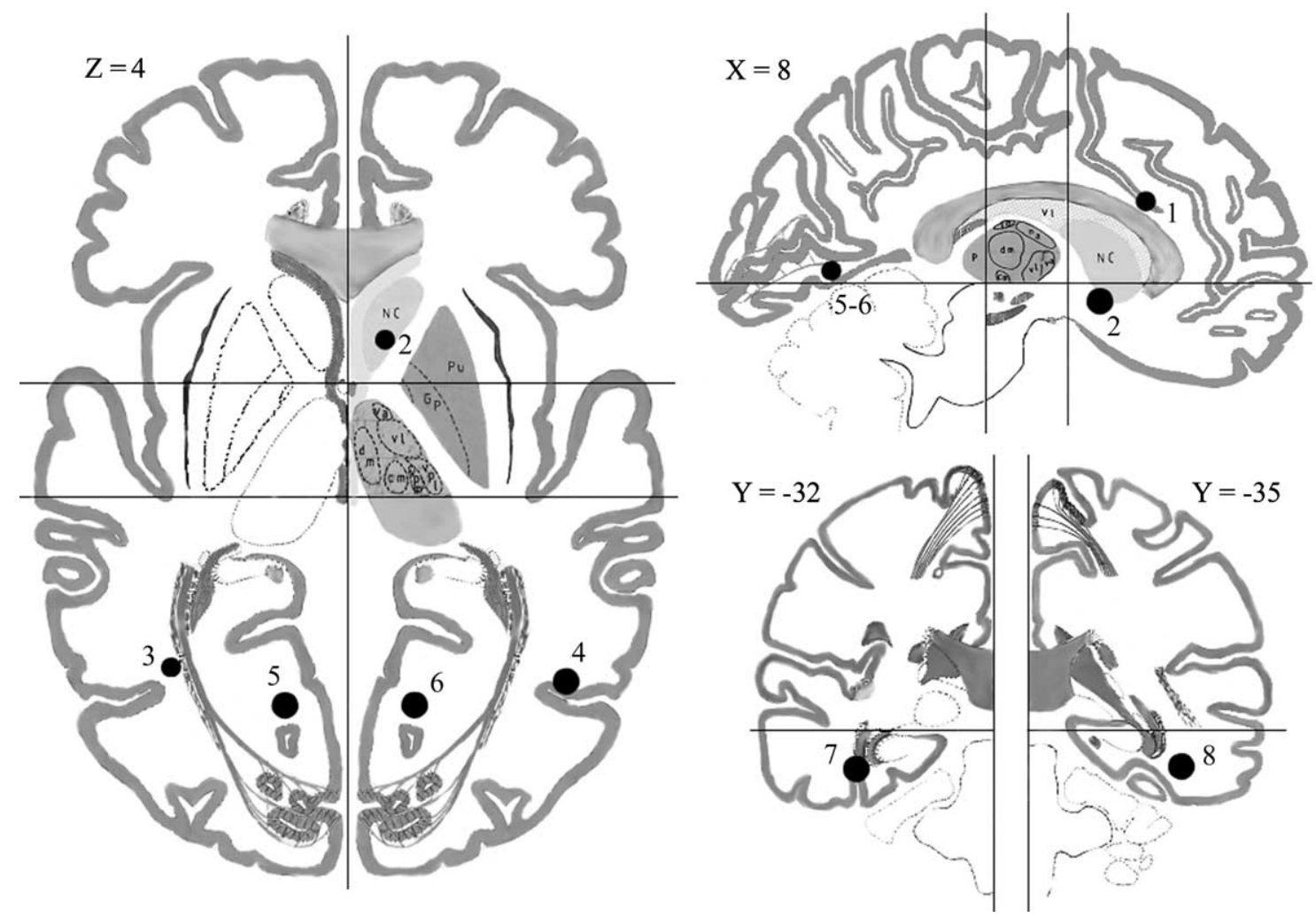

Fig. 4. The dipole locations plotted in the slices of Talairach Atlas. Sagittal plane $(x=8)$ shows the location of dipole 2 with a large circle, and in addition dipoles 1 and 5-6 (small circles) are seen because of their proximity to the midline. Transverse plane $(z=4)$ shows the location of dipoles $4-6$ with large circles, and in addition the dipoles 2 and 3 (small circles) located in the neighbor slices are seen. Coronal plane shows locations of the dipoles 7 ( $y=-32$ ) and $8(y=-35)$. 
sources 1-4 (Fig. 3d). The significant differences for SP-DP pair were found mainly in dipoles 1 and 2 .

\section{Discussion}

The purpose of this study was to create a dipole-source model for familiar face recognition and comparison. During the same session, the participants compared pairs of faces while ignoring patterns (Face task) and pairs of patterns while ignoring faces (Pattern task). The face images were photographs of people personally known to the participants, which made them highly relevant and more emotionally arousing than famous or learned-during-experiment faces. When the attention of a participant was directed to the face comparison, the white dots were considered as an additional noise. Thus, the results from the Face task, unlike the Pattern task, can be considered separately. The reaction times to same person's faces were shorter (priming effect) than to non-matching faces; similar results were reported for the categorical comparison of non-familiar faces (Munte et al., 1998). The priming effect in the brain electrical activity was manifested mainly in the amplitude reduction of the N400-like components in the ERPs elicited by the pairs of photographs of the same person as compared to the pairs of pictures showing different persons.

Our models are compatible with the well-known models based on psychological (Bruce and Young, 1986) or functional magnetic resonance imaging (fMRI) and positron emission tomography (PET) (Haxby et al., 2000) data for face processing. Of course, the point-size dipole locations provide only a rough estimate for the active brain regions and cannot precisely show the real geometry of the active area. However, our models were able to dissociate the components in the occipital cortex (the dipoles 5 and 6 explain components at 120 and $180 \mathrm{~ms}$ ) and in the inferotemporal cortex (the dipoles 7 and 8 explain components at 150-160 ms). Similar locations for the sources of P120 and N170 were suggested in a magnetoencephalography/EEG research (Linkenkaer-Hansen et al., 1998). The inferotemporal activity has been recorded in PET and fMRI studies of face processing (Haxby et al., 2000); this area is supposed to be the source for the facespecific ERP components (Allison et al., 1994; Bentin et al., 1996). Dipolar sources in the IT cortex were also modeled by Watanabe et al. (1999).

Our models revealed hemispheric asymmetries on the earlier stages of face processing (components at 120 and $150-170 \mathrm{~ms}$ ). Shorter latencies of face-specific N170 in the right hemisphere were reported by other authors (Yovel et al., 2003). The early match-mismatch effects in the Face task observed in the scalp-recorded ERPs in 200-250 ms (Mnatsakanian and Tarkka, 2003) can be explained by differences in the activity of the sources 2, 4, 5, and 8 in our models. The difference in 150-170 ms was significant for the activity of the source 7 (left IT cortex), while it was overlooked in scalp ERP analysis. The early priming effects were reported at about $200 \mathrm{~ms}$ for the categorical comparison of non-familiar faces (Munte et al., 1998), and for earlier latencies in the studies with face and shape repetition (George et al., 1997).

The brain activity after $250 \mathrm{~ms}$ was explained mainly by the dipoles 1-4 having temporal and frontal locations. The preparatory activity in the supplementary motor area and the activation of primary motor cortex seem to be quite small during the analysis period and probably have minor effects (the mean RT values in our study were about $800 \mathrm{~ms}$ ). The sources for the components with latencies of about $400 \mathrm{~ms}$ in face/word tasks have been located by other authors to be in the temporal and frontal cortices (Halgren et al., 1994a,b). Wide, mostly temporal, networks are implicated in the metabolic studies as sources for personal identity-related brain activity (Haxby et al., 2000; Leveroni et al., 2000; Tempini et al., 1998). It is possible that our temporal sources (dipoles 3 and 4) reflect such activity, at least partially. The SWFs obtained by the application of the base model to Pattern task showed that the activity was less in these sources than in the Face task. It seems that the comparison of familiar faces elicits more activity in these regions than seeing a familiar face on the background. However, the influence of the familiarity of the face cannot be defined in this study.

The major differences in SWFs between the DF and SF conditions were seen in 350-600 $\mathrm{ms}$ in dipoles $1-2$, located close to the midline, in the vicinity of the anterior cingulate cortex (ACC). The ACC is a part of the brain's limbic system. A number of studies dealing with ACC have proposed a role in the conflict monitoring (conflict between competing information streams), executive control or performance monitoring, error detection and correction, response selection, emotional and attentional regulation (Bush et al., 2000). Extensive evidence suggests that ACC exhibits anatomical and functional specificity; separate areas of ACC are involved in cognition and emotion (Bush et al., 2000; Rossell et al., 2001). The activation of distinct regions of ACC was observed in fMRI studies of semantic priming of words (Rossell et al., 2001) and in the delayed face recognition task (Druzgal and D'Esposito, 2001). In the latter study the activity in ACC was greater when the probe face did not match the remembered face. This has a resemblance to our experiment, where the dipole 1 (located in ACC) was stronger for DF than for SF, while both showed similar temporal behavior. The dipole 2 had a prominent peak at $300 \mathrm{~ms}$, similar for both conditions, and another peak at $400 \mathrm{~ms}$ observed only in DF. In the study presenting the dipole models for completing familiar faces with congruous and incongruous parts (Jemel et al., 1999), despite differences in locations and orientations, the two dipoles in the anterior areas showed in their activity dynamics some similarities to the frontal dipoles in our models. Unlike neuroimaging studies, dipole models cannot indicate the exact anatomical locations; thus we can only 
assume that our frontal dipoles reflect the activity of distinct regions of ACC, as well as the activity of some other areas in the vicinity. For the dipole 2, this can be caudate nucleus as well as some neighboring structures (nucleus accumbens, putamen, etc.). It can be speculated that the similar components may reflect the activity common for both conditions, like emotional arousal due to the face familiarity. The frontal dipoles 1 and 2 show clear matchmismatch differences; however, the specificity of these differences is not clear. The SWFs obtained for Pattern task showed that the activity of both sources 1 and 2 was also task-dependent, and the match-mismatch distinctions were different from those observed in Face task. The dipole 1 had similar dynamics in both tasks, while a smaller amplitude for Pattern task. The SWFs of source 2 had peaks around 300 and $400 \mathrm{~ms}$ for both SP and DP conditions, and SP-DP differences were seen for both peaks. Thus, the specificity of the face recognition and comparison cannot yet be definitely answered by analyzing also the Pattern task. It may well be that there are several N400-like components.

The models proposed for recognition and comparison of familiar faces explain about $97 \%$ of grand average ERP and about $90 \%$ of individual data and show a clear temporal and spatial separation of active sources. These sources can characterize the activity presumably common for both conditions (like the recognition of a familiar person); in addition, the differential activity related to the match/mismatch item processing can be determined by these sources.

\section{References}

Allison T, Ginter H, McCarthy G, Nobre AC, Puce A, Luby M, Spencer DD. Face recognition in human extrastriate cortex. J Neurophysiol 1994;71:821-5.

Barrett SE, Rugg MD. Event-related potentials and the semantic matching of pictures. Brain Cogn 1990;14:201-12.

Bentin S, Allison T, Puce A, McCarthy G. Electrophysiological studies of face perception in humans. J Cogn Neurosci 1996;8:551-65.

Berg P, Scherg M. A fast method for forward computation of multiple-shell spherical head models. Electroenceph clin Neurophysiol 1994;90: 58-64.

Bruce V, Young A. Understanding face recognition. Br J Psychol 1986;77: 305-27.

Bush G, Luu P, Posner MI. Cognitive and emotional influences in anterior cingulate cortex. Trends Cogn Sci. 2000;4:215-22.

Druzgal TJ, D’Esposito M. A neural network reflecting decisions about human faces. Neuron 2001;32:947-55.
Eimer M. Event-related brain potentials distinguish processing stages involved in face perception and recognition. Clin Neurophysiol 2000; 111:694-705.

George N, Jemel B, Fiori N, Renault B. Face and shape repetition effects in humans: a spatio-temporal ERP study. NeuroReport 1997;8:1417-23.

Halgren E, Baudena P, Heit G, Clarke JM, Marinkovic K. Spatio-temporal stages in face and word processing. 1. Depth recorded potentials in the human occipital, temporal and parietal cortices. J Physiol Paris 1994a; $88: 1-50$.

Halgren E, Baudena P, Heit G, Clarke JM, Marinkovic K, Chauvel P. Spatio-temporal stages in face and word processing. 2. Depth recorded potentials in the human frontal and Rolandic cortices. J Physiol Paris 1994b;88:51-80.

Haxby JV, Hoffman EA, Gobbini MI. The distributed human neural system for face perception. Trends Cogn Sci 2000;4:223-32.

Jemel B, George N, Olivares E, Fiori N, Renault B. Event-related potentials to structural face incongruity processing. Psychophysiology 1999;36: 437-52.

Kutas M, Hillyard SA. Event-related brain potentials to semantically inappropriate and surprisingly large words. Biol Psychol 1980;11:99-116.

Leveroni CL, Seidenberg M, Mayer AR, Mead LA, Binder JR, Rao SM. Neural systems underlying the recognition of familiar and newly learned faces. J Neurosci 2000;20:878-86.

Linkenkaer-Hansen K, Palva JM, Sams M, Hietanen JK, Aronen HJ, Ilmoniemi RJ. Face-selective processing in human extrastriate cortex around $120 \mathrm{~ms}$ after stimulus onset revealed by magneto- and electroencephalography. Neurosci Lett 1998;253:147-50.

McPherson WB, Holcomb PJ. An electrophysiological investigation of semantic priming with pictures of real objects. Psychophysiology 1999; 36:53-65.

Mnatsakanian EV, Tarkka IM. Matching of familiar faces and abstract patterns: behavioral and high-resolution ERP study. Int J Psychophysiol 2003;47:217-27.

Munte TF, Brack M, Grootheer O, Wieringa BM, Matzke M, Johannes S. Brain potentials reveal the timing of face identity and expression judgments. Neurosci Res 1998;30:25-34.

Rossell SL, Bullmore ET, Williams SC, David AS. Brain activation during automatic and controlled processing of semantic relations: a priming experiment using lexical-decision. Neuropsychologia 2001;39:1167-76.

Rossion B, Campanella S, Gomez CM, Delinte A, Debatisse D, Liard L, Dubois S, Bruyer R, Crommelinck M, Guerit JM. Task modulation of brain activity related to familiar and unfamiliar face processing: an ERP study. Clin Neurophysiol 1999;110:449-62.

Talairach JA, Tournoux P. Co-planar stereotaxic atlas of the human brain. New York: Thieme Verlag; 1988.

Tempini MLG, Price CJ, Josephs O, Vanderberhe R, Cappa SE, Kapur N, Frackowiak RSJ. The neural systems sustaining face and proper-name processing. Brain 1998;121:2103-18.

Watanabe S, Kakigi R, Koyama S, Kirino E. Human face perception traced by magneto- and electro-encephalography. Cogn Brain Res 1999;8: $125-42$.

Yovel G, Levy J, Grabowecky M, Paller KA. Neural correlates of the leftvisual-field superiority in face perception appear at multiple stages of face processing. J Cogn Neurosci 2003;15:462-747. 